\title{
A founder haplotype of APOE-Sendai mutation associated with lipoprotein glomerulopathy
}

\author{
Kentaro Toyota ${ }^{1}$, Taeko Hashimoto ${ }^{1}$, Daisuke Ogino ${ }^{1}$, Akira Matsunaga ${ }^{1}$, Minoru Ito $^{2}$, Ikuto Masakane ${ }^{3}$, \\ Noriyuki Degawa ${ }^{4}$, Hiroshi Sato ${ }^{5}$, Sayuri Shirai ${ }^{6}$, Kazuo Umetsu ${ }^{7}$, Gen Tamiya ${ }^{8}$, Takao Saito ${ }^{9}$ \\ and Kiyoshi Hayasaka ${ }^{1}$
}

\begin{abstract}
Lipoprotein glomerulopathy (LPG) is a hereditary disease characterized by lipoprotein thrombi in the glomerulus, hyperlipoproteinemia, and a marked increase in serum apolipoprotein E (APOE). More than 12 APOE mutations have been identified as causes of LPG, and APOE-Sendai (Arg145Pro) mutation was frequently detected in patients from the eastern part of Japan including Yamagata prefecture. Recently, effective therapy with intensive lipid-lowering agents was established, and epidemiologic data are required for early diagnosis. We determined the haplotype structure of $A P O E$-Sendai in 13 patients from 9 unrelated families with LPG, and found that the haplotype of all APOE-Sendai mutations was identical, suggesting that $A P O E-S e n d a i$ mutation is common in Japanese patients probably through a founder effect. We also studied the gene frequency of APOE-Sendai in 2023 control subjects and 418 patients receiving hemodialysis in Yamagata prefecture using the TaqMan method, but did not identify any subjects carrying the mutation, indicating that it is very rare in the general population even in the eastern part of Japan. In addition to $A P O E$ mutation, other genetic and/or epigenetic factors are considered to be involved in the pathogenesis of LPG because of its low penetrance. The patients did not have a common haplotype of the counterpart $A P O E$ allele, and some patients had the same haplotype of the counterpart $A P O E$ allele as the asymptomatic carriers. These results suggest that the counterpart $A P O E$ allele is not likely associated with the onset of LPG. Further study is required to clarify the pathogenesis of LPG.
\end{abstract}

Journal of Human Genetics (2013) 58, 254-258; doi:10.1038/jhg.2013.8; published online 14 February 2013

Keywords: $A P O E ; A P O E-S e n d a i ;$ lipoprotein glomerulopathy

\section{INTRODUCTION}

Lipoprotein glomerulopathy (LPG) is a rare hereditary disease characterized by lipoprotein thrombi in the intraglomerular capillaries, an abnormal plasma lipoprotein profile similar to type III hyperlipoproteinemia and a marked increase in serum apolipoprotein E (APOE). ${ }^{1}$ The group of Saito ${ }^{2}$ first described a patient with LPG in 1989, and finally identified the cause of LPG as APOE mutation in 1997. ${ }^{3}$ More than 100 patients have been reported worldwide, but the majority were Japanese and Chinese. ${ }^{1,4-9}$ In most cases, the first clue to diagnosis is the incidental discovery of proteinuria or development of signs and symptoms suggestive of nephrotic syndrome, which ultimately progresses to chronic renal failure. Ordinary treatments for nephrotic syndrome including steroids, immunosuppressants and anticoagulants were ineffective. Kidney transplantation has been performed; however, LPG reportedly recurred in transplanted grafts. ${ }^{1}$ Recently, therapy with intensive lipid-lowering agents including fibrates was reported to result in clinical remission accompanying histological resolution. $5,10,11$ The establishment of effective therapy will promote the early detection of LPG to avoid chronic renal failure, which requires epidemiologic data.

APOE mutations causing LPG were initially reported to be located around low-density lipoprotein (LDL) receptor-binding domains, but mutations located in regions other than the receptor domain have been reported. ${ }^{1}$ To date, more than 12 mutations have been reported, ${ }^{4,7,8}$ and APOE-Sendai (Arg145Pro) mutation is common in Japan, especially in the eastern part. ${ }^{13,5,6,10}$

In the present research, we studied the haplotype of APOESendai in 13 Japanese patients from 9 unrelated families with LPG, and confirmed the common haplotype, suggesting that the frequency of APOE-Sendai mutation in Japanese patients is probably through a founder effect. We also studied the gene frequency of APOE-Sendai in control subjects and patients receiving hemodialysis due to renal failure using the TaqMan method, but did not identify any subjects carrying the mutation, indicating that it

${ }^{1}$ Department of Pediatrics, Yamagata University School of Medicine, Yamagata, Japan; ${ }^{2}$ Dialysis Center, Yabuki Hospital, Yamagata, Japan; ${ }^{3}$ Yabuki Shima Clinic, Yamagata, Japan; ${ }^{4}$ Department of Internal Medicine, Yamagata City Hospital Saiseikan, Yamagata, Japan; ${ }^{5}$ Graduate School of Pharmaceutical Sciences, Tohoku University, Sendai, Japan; ${ }^{6}$ Division of Nephrology and Hypertension, Department of Internal Medicine, St Marianna University School of Medicine, Miyamae-ku, Kawasaki, Japan; ${ }^{7}$ Department of Legal Medicine, Yamagata University School of Medicine, Yamagata, Japan; ${ }^{8}$ Advanced Molecular Epidemiology Research Institute, Yamagata University School of Medicine, Yamagata, Japan and ${ }^{9}$ General Medical Research Center, Fukuoka University School of Medicine, Fukuoka, Japan

Correspondence: Professor K Hayasaka, Department of Pediatrics, Yamagata University School of Medicine, 2-2-2 lida-nishi, Yamagata, Japan.

E-mail: hayasaka@med.id.yamagata-u.ac.jp

Received 5 October 2012; revised 8 January 2013; accepted 23 January 2013; published online 14 February 2013 
is a very rare mutation even in Yamagata prefecture, in the eastern part of Japan.

\section{SUBJECTS AND METHODS}

The Ethics Committee of the Yamagata University School of Medicine approved our study.

\section{Patients with LPG}

We enrolled 13 patients with LPG from 9 unrelated Japanese families who were diagnosed by renal biopsy from age 7 to 79 years. Some of them were previously reported. After informed consent was obtained from the patients, genomic DNA was isolated from the peripheral blood.

\section{Patients receiving hemodialysis in Yamagata prefecture}

From September 2011 to January 2012, we recruited 418 patients (male-tofemale ratio, 240:178; ages from 23 to 93 years; median age, 78 years) who received hemodialysis with no definite diagnosis of LPG. Causes of renal insufficiency were as follows: diabetic nephropathy $(n=144)$, chronic glomerulonephritis $(n=106)$, renal sclerosis $(n=23)$, primary nephrotic syndrome $(n=16)$, polycystic kidney disease $(n=14)$, rapidly progressive glomerulonephritis $(n=12)$, malignant neoplasm $(n=8)$, urinary tract malformation $(n=8)$, collagen disease $(n=6)$, nephropathy of pregnancy $(n=4)$, familial cystinuria $(n=2)$, acute heart failure $(n=2)$, hepatorenal syndrome $(n=2)$, renal amyloidosis $(n=1)$, Alport syndrome $(n=1)$, renal coloboma syndrome $(n=1)$, mitochondrial disorder $(n=1)$, urate nephropathy $(n=1)$ and unknown causes $(n=66)$. After informed consent was obtained from the patients, genomic DNA was isolated from the peripheral blood.

\section{Control subjects for population studies in the eastern part of Japan, Yamagata prefecture}

From August 2010 to September 2012, 2023 consecutive newborns (males: 1021, females: 1002) in Yamagata prefecture of Japan were screened for APOE-Sendai mutation. After informed consent was obtained from the parents, genomic DNA was isolated from spare dried blood spots from neonatal screening cards.

\section{DNA extraction}

We prepared genomic DNA from peripheral blood using Quick Gene 610L (Fuji Film, Tokyo, Japan) according to the manufacturer's instructions and from spare dried blood spots from neonatal screening cards using a method previously reported. ${ }^{12}$

\section{APOE mutation analysis}

All coding exons and exon-intron boundaries of $A P O E$ were amplified using the primer sets shown in Figure 1 and Supplementary Table 1. Sequencing reaction was performed using the BigDye Terminator v. 1.1 Cycle Sequencing Ready Reaction Kit (PE Applied Biosystems, Foster City, CA, USA), with PCR and sequence primers described in Supplementary Table 1. After the purification of sequencing products, the sequence was determined on an ABI $3500 \mathrm{XI}$ Genetic analyzer (PE Applied Biosystems).

\section{Establishment of method to detect APOE-Sendai mutation}

Genotype determination of APOE-Sendai (c.488 G >C) was performed using TaqMan SNP Genotyping Assays (PE Applied Biosystems). Two TaqMan probes, each specific for one of the available alleles (wild-type probe labeled with VIC fluorescence: 5'-CAAGCTGCGTAAGCG-3', APOE-Sendai-specific probe labeled with FAM fluorescence: 5'-CAAGCTGCCTAAGCG-3') were present in the reaction, specifically annealing to the target region between the two primers (F-primer: 5'-CCTCGCCTCCCACCTG-3', R-primer: $5^{\prime}$-CGGCATCGCGGAGGA- $3^{\prime}$ ). The assay design and conditions were based on the allelic discrimination protocol from Applied Biosystems.

\section{Haplotype analysis}

We determined 26 single-nucleotide polymorphisms (SNPs) of APOE including 23 SNPs described by Fullerton et al. ${ }^{13}$ one SNP located at the site of
APOE-Sendai and two SNPs located downstream of APOE, based on the data of GenBank accession number NC_000019.9 (Figure 1). At first, we amplified two overlapping fragments: fragment $1(4.6 \mathrm{~kb})$ including exons 1 to 4 (45408004 to 45412689$)$ using primers $5 \mathrm{~F}$ and $5 \mathrm{R}$, and fragment $2(4.4 \mathrm{~kb})$ including exon 4 and downstream of APOE (45411653 to 45416067) using primers $6 \mathrm{~F}$ and $6 \mathrm{R}$ (Supplementary Table 1). Then, we performed allelespecific oligonucleotide PCR (ASO-PCR) and separated the allele carrying $A P O E-S e n d a i$ and that carrying wild-type $A P O E$ in each individual. For the ASO-PCR of the $5^{\prime}$ part of $A P O E$, we used fragment 1 as a template with the four different combinations of primer sets: 471A-F: 5'-CCTCAGCCTCCC AAGTAGCTA-3'， 471G-F: 5'-CTCAGCCTCCCAAGTAGCTG-3'， 4037C-R (wild type): 5'-GCGGAGGAGCCGCTTAC-3', 4037G-R (APOE-Sendai): $5^{\prime}$-GCGGAGGAGCCGCTTAG-3'. For ASO-PCR of the $3^{\prime}$ part of APOE, we used fragment 2 as a template with four different combinations of primer sets: 4037G-F (wild-type): 5'-CACCTGCGCAAGCTGCG-3'， 4037C-F (APOESendai): 5'-CACCTGCGCAAGCTGCC-3' ${ }^{\prime}$ 7636G-R: 5'-AAATTTAAAAAAGG

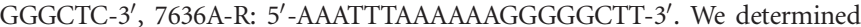
the SNPs by sequencing the products of ASO-PCR using the primers in Supplementary Table 2 .

\section{RESULTS}

Mutation analysis in patients with LPG

We determined the sequence of $A P O E$ in 13 patients and family members, and confirmed that all patients and several asymptomatic family members were heterozygotes of APOE-Sendai mutation (Figure 2).

\section{Haplotype analysis}

We determined 26 SNPs of APOE including 23 SNPs described by Fullerton et al. ${ }^{13}$ one SNP determined at a site of APOE-Sendai and two SNPs located downstream of APOE based on the GenBank accession number NC_000019.9 (Figure 1 and Table 1). We classified the haplotype according to the report of Fullerton et al. ${ }^{13}$ As shown in Figure 2 and Table 1, the haplotype of all alleles carrying APOESendai mutation was identical and was likely derived from haplotype 1 of 83 . The APOE-Sendai mutation is common among unrelated patient populations in the eastern part of Japan, which are probably shared because of some stochastic population process such as a founder effect in a founder population. As for the counterpart APOE allele, the haplotype of counterpart APOE alleles in 13 LPG patients was not identical or similar (Figure 2), and the patient (II-3) in family 1 and the patient (II-1) in family 2 had the same haplotype of the counterpart allele as that of the asymptomatic brother (II-1) in family 1 and that of the asymptomatic father (I-1) and younger sister (II-3) in family 2, respectively. These suggest that the counterpart APOE allele is not likely associated with the pathogenesis.

\section{Establishment of detection method of APOE-Sendai mutation} For the population study of the APOE-Sendai mutation, we established a simple method to detect the mutation using the TaqMan method, as described in the Subjects and methods. We checked the sensitivity and efficacy by studying all patients and 100 control specimens, and confirmed the complete sensitivity and efficacy (data not shown).

\section{APOE-Sendai mutation in patients receiving hemodialysis in Yamagata prefecture}

To study the frequency of LPG due to APOE-Sendai in patients with renal insufficiency in Yamagata prefecture, we screened for APOE-Sendai in 418 patients with renal insufficiency not due to apparent LPG using the TaqMan method, and identified no mutation in the patients. 


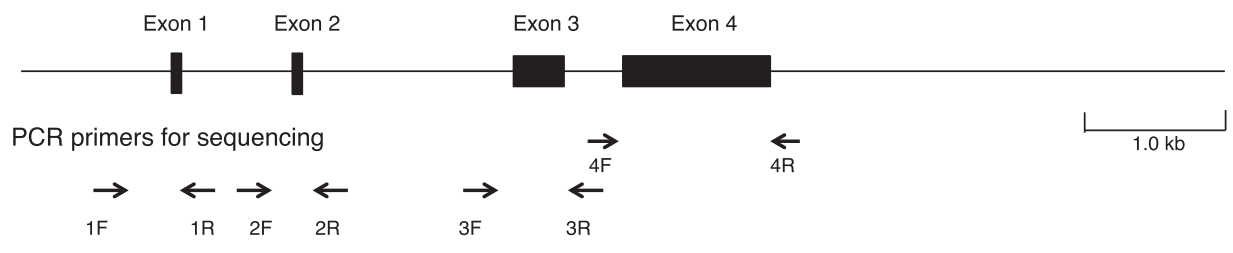

Primers for haplotype determination $1^{\text {st }} \mathrm{PCR}$

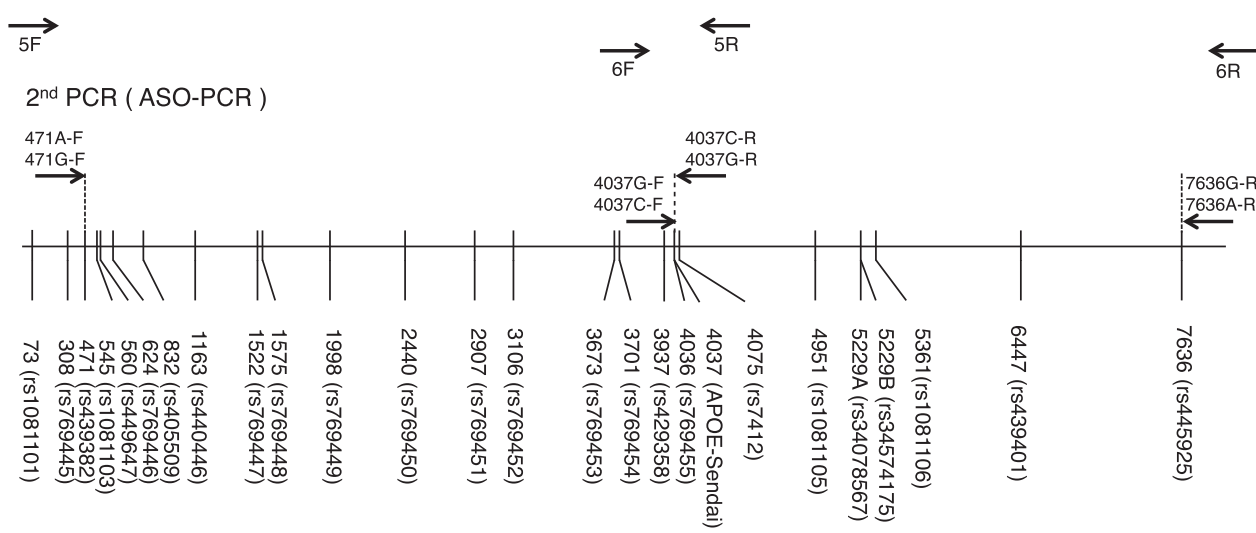

Figure 1 Structure and position of single-nucleotide polymorphisms (SNPs) and PCR primers of apolipoprotein $E$ (APOE). The nucleotide numbers are according to Fullerton et al. ${ }^{13}$

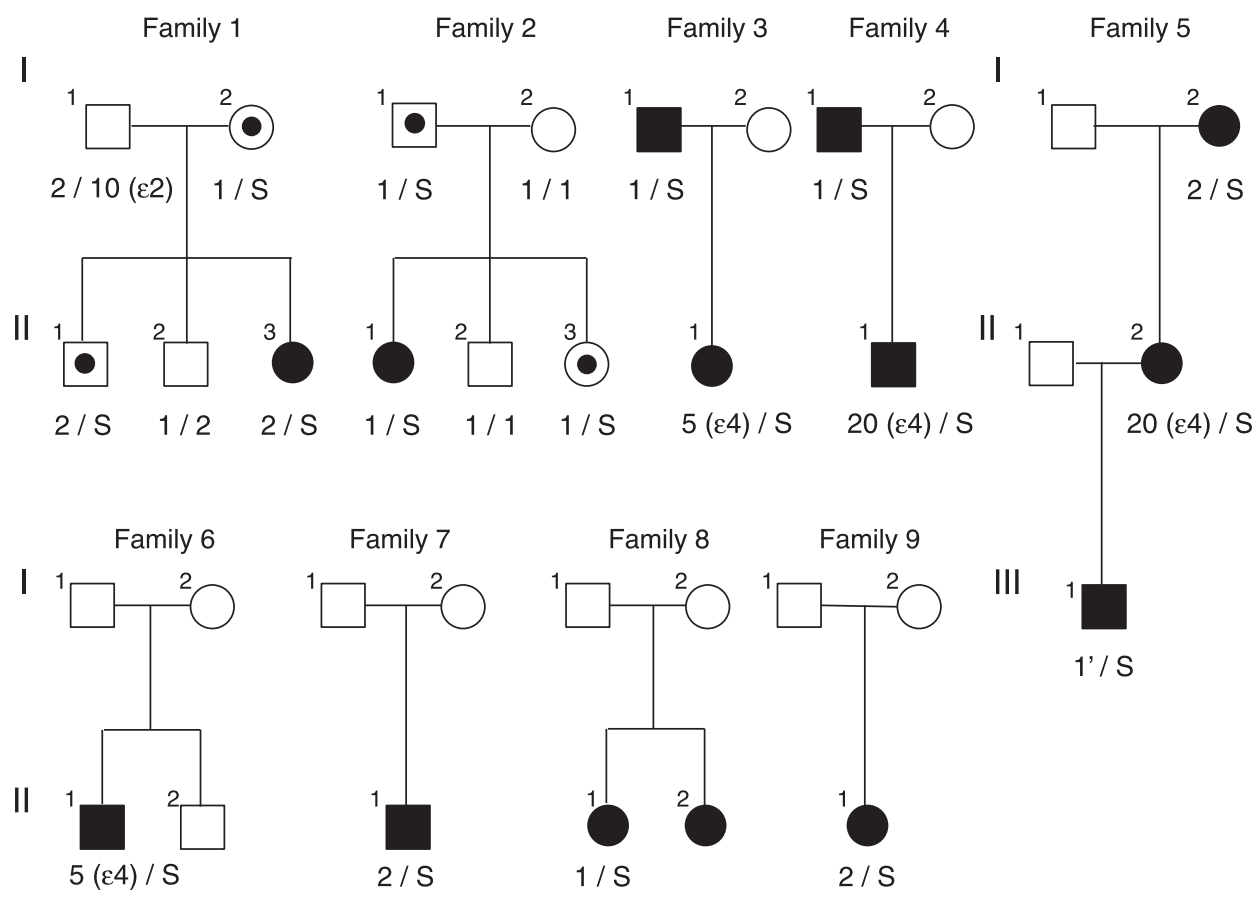

Figure 2 Family pedigrees and haplotypes of apolipoprotein $E$ ( $A P O E$ ). The number under each symbol represents the haplotype of $A P O E$. Characters in parentheses indicate $\varepsilon 2$ and $\varepsilon 4$ alleles, which encode APOE2 and APOE4, respectively. Numbers without parentheses belong to the $\varepsilon 3$ allele encoding APOE3. S and 1' indicate a haplotype of APOE-Sendai and a derivative of haplotype 1 that shows $1163 \mathrm{C}>\mathrm{G}$ (rs440446) polymorphism, respectively.

\section{Population studies of APOE-Sendai in Yamagata prefecture} We screened for APOE-Sendai in 2023 newborns in Yamagata prefecture using the TaqMan method, and did not identify any newborns carrying APOE-Sendai.

\section{DISCUSSION}

We determined the haplotype of APOE-Sendai in 13 patients from 9 unrelated families with LPG, and found that the haplotype of the mutant allele was identical, suggesting that APOE-Sendai mutation is common in patients from the eastern part of Japan including Yamagata prefecture, probably through a founder effect. We also studied the APOE-Sendai frequency in control subjects and patients receiving hemodialysis in Yamagata prefecture, and did not identify any subjects carrying the mutation, indicating that $A P O E$ Sendai is very rare in the general population even in the eastern part of Japan. 
Table 1 Haplotype of $\varepsilon 3$ group and APOE-Sendai

Site

\begin{tabular}{|c|c|c|c|c|c|c|c|c|c|c|c|c|c|c|c|c|}
\hline Haplotype $^{a}$ & 308 & 545 & 560 & 624 & 832 & 1163 & 1522 & 1575 & 1998 & 2440 & 2907 & 3673 & 3701 & 4037 & 5361 & Population counts ${ }^{\mathrm{a}}$ \\
\hline 26 & C & C & $T$ & $\mathrm{C}$ & $\mathrm{T}$ & C & $\mathrm{G}$ & C & $\mathrm{G}$ & $\mathrm{G}$ & $\mathrm{T}$ & C & - & $\mathrm{G}$ & $\mathrm{T}$ & 1 \\
\hline 21 & C & C & $A$ & C & $\mathrm{T}$ & C & $\mathrm{G}$ & $T$ & $\mathrm{G}$ & $\mathrm{G}$ & $\mathrm{T}$ & C & - & $\mathrm{G}$ & $\mathrm{T}$ & 1 \\
\hline 4 & C & C & $\mathbf{T}$ & $\mathrm{T}$ & $\mathrm{T}$ & C & $\mathrm{G}$ & C & G & $\mathrm{G}$ & $\mathrm{T}$ & C & - & $\mathrm{G}$ & $\mathrm{T}$ & 16 \\
\hline 29 & C & C & $A$ & $\mathrm{~T}$ & $\mathrm{~T}$ & C & G & C & G & G & $\mathrm{T}$ & C & CT & G & $\mathrm{T}$ & 1 \\
\hline 1 & C & C & $A$ & $\mathrm{~T}$ & $\mathrm{~T}$ & C & G & C & G & G & $\mathrm{T}$ & C & - & G & $\mathrm{T}$ & 45 \\
\hline APOE-Sendai & C & C & A & $\mathrm{T}$ & $\mathrm{T}$ & $C$ & $\mathrm{G}$ & C & G & G & $\mathrm{T}$ & C & - & C & $\mathrm{T}$ & \\
\hline 30 & C & $\mathrm{C}$ & $A$ & $\mathrm{~T}$ & $\mathrm{~T}$ & $C$ & $\mathrm{G}$ & $C$ & $\mathrm{G}$ & G & G & $C$ & - & $\mathrm{G}$ & $\mathrm{T}$ & 1 \\
\hline 14 & C & C & $A$ & $\mathrm{~T}$ & $\mathrm{~T}$ & C & A & $C$ & $\mathrm{G}$ & $\mathrm{G}$ & $\mathrm{T}$ & C & - & G & $\mathrm{C}$ & 2 \\
\hline 19 & C & C & A & $\mathrm{T}$ & $\mathbf{G}$ & C & G & C & $\mathrm{G}$ & $\mathrm{G}$ & $\mathrm{T}$ & C & - & G & $\mathrm{T}$ & 1 \\
\hline 6 & C & C & A & $\mathrm{T}$ & $\mathbf{G}$ & G & $\mathrm{G}$ & C & $\mathrm{G}$ & $\mathrm{G}$ & $\mathrm{T}$ & G & - & $\mathrm{G}$ & $\mathrm{T}$ & 8 \\
\hline 27 & $\mathbf{T}$ & C & $\mathbf{T}$ & $\mathrm{T}$ & $\mathbf{G}$ & G & $\mathrm{G}$ & C & $\mathrm{G}$ & $\mathrm{G}$ & $\mathrm{T}$ & C & - & G & $\mathrm{T}$ & 1 \\
\hline 8 & C & C & $\mathbf{T}$ & $\mathrm{T}$ & $\mathbf{G}$ & G & $\mathrm{G}$ & C & $\mathrm{G}$ & $\mathrm{G}$ & $\mathrm{T}$ & C & - & $\mathrm{G}$ & $\mathrm{T}$ & 5 \\
\hline 3 & C & C & $\mathbf{T}$ & $\mathrm{T}$ & $\mathbf{G}$ & $\mathbf{G}$ & $\mathrm{G}$ & C & $\mathrm{G}$ & $A$ & $\mathrm{~T}$ & C & - & G & $\mathrm{T}$ & 15 \\
\hline 11 & C & $C$ & A & C & $\mathbf{G}$ & $\mathbf{G}$ & $\mathrm{G}$ & C & G & A & $\mathrm{T}$ & C & - & $\mathrm{G}$ & $\mathrm{T}$ & 2 \\
\hline 2 & $C$ & $C$ & A & $\mathrm{T}$ & $\mathbf{G}$ & $\mathbf{G}$ & $\mathrm{G}$ & $C$ & $\mathrm{G}$ & A & $\mathrm{T}$ & $C$ & - & $\mathrm{G}$ & $\mathrm{T}$ & 43 \\
\hline 28 & $C$ & $\mathbf{T}$ & A & $\mathrm{T}$ & $\mathbf{G}$ & $\mathbf{G}$ & $\mathrm{G}$ & $C$ & $\mathrm{G}$ & A & $\mathrm{T}$ & $C$ & - & $\mathrm{G}$ & $\mathrm{T}$ & 1 \\
\hline 7 & C & C & $A$ & $\mathrm{~T}$ & $\mathbf{G}$ & $\mathbf{G}$ & $\mathrm{G}$ & C & $\mathrm{G}$ & A & $\mathrm{T}$ & $\mathrm{C}$ & - & $\mathrm{G}$ & $\mathrm{T}$ & 8 \\
\hline 25 & $C$ & $C$ & $A$ & $\mathrm{~T}$ & G & G & $\mathrm{G}$ & $C$ & $A$ & $A$ & $\mathrm{~T}$ & C & - & $\mathrm{G}$ & $\mathrm{T}$ & 1 \\
\hline
\end{tabular}

Bold characters represent minor alleles among $\varepsilon 3$ group.

a Classification of haplotype, population counts and number of single-nucleotide polymorphisms (SNPs) and mutation sites described by Fullerton et al. ${ }^{13}$

APOE, a 34-kDa protein consisting of 299 amino acids, plays a significant role in the metabolism of lipids and lipoproteins. ${ }^{1}$ It is a structural component of chylomicron, chylomicron remnants, very low-density lipoproteins, and a subgroup of high-density lipoproteins and serves as a ligand for LDL and APOE receptors. ${ }^{1}$ The APOE gene is located on chromosome $19 \mathrm{q} 13.2$ and has three common alleles, $\varepsilon 2$ (0.02 of frequency in Japan), $\varepsilon 3$ ( 0.87 of frequency) and $\varepsilon 4$ ( 0.11 of frequency), that encode APOE2 (Arg158Cys), APOE3 and APOE4 (Cys112Arg), respectively. Whereas APOE3 and APOE4 are thought to bind equally to the LDL receptors, APOE2 is defective in its lipoprotein receptor binding, and homozygosity for APOE2 results in the development of type III hyperlipoproteinemia. ${ }^{1}$ Most APOE variants causing LPG are located in the LDL receptor and are also frequently associated with hyperlipoproteinemia similar to type III. APOE-Sendai (Arg145Pro), the first APOE mutation in LPG, substitutes proline for arginine in the LDL receptor binding domain. Moreover, APOE-Chicago (Arg147Pro) ${ }^{14}$ and APOE-Guangzhou (Arg150Pro $)^{15}$ have been reported in the United States and China, respectively. Proline acts as a structural disrupter in the middle of the $\alpha$-helix, and is commonly found as the first residue of the $\alpha$-helix or in turns in an amino acid arrangement. ${ }^{1}$ Accordingly, proline is not in or around the LDL receptor binding domain of APOE, which forms a continuous $\alpha$-helix. Once the substitution of proline occurs in this area, however, APOE may markedly change its structure, aggregate lipoproteins and induce intraglomerular lipoprotein thrombi of LPG.

More than 12 APOE mutations associated with LPG have been reported so far. ${ }^{4,7,8}$ In contrast to the mutations in a single or a few families, ${ }^{1,4,7,8,14-17}$ APOE-Sendai (Arg145Pro), APOE-Kyoto (Cys25Arg) and APOE Tokyo-Maebashi (Leu141_Lys143del or Arg142_Leu144del) are prevalent mutations. ${ }^{1-3,5,6,9,10,18-23}$ APOESendai is common in Japan, and its distribution is restricted to the eastern part of Japan. ${ }^{1-3,5,6,10}$ To clarify the mechanism of its commonality, we determined the haplotype of APOE-Sendai detected in unrelated families, and found that the APOE-Sendai haplotype was identical and likely derived from one of the subtypes of APOE3 (haplotype 1 in the classification by Fullerton et al. ${ }^{13}$ ) (Table 1). This suggests that the APOE-Sendai mutation spread from one founder mutation. We speculated that the APOE-Sendai mutation was generated less than 80000 years ago when haplotype 1 of APOE was generated. ${ }^{13}$ On the contrary, APOE-Kyoto and APOE TokyoMaebashi are also common and have been detected worldwide. . $^{1,4,9,18-23}$ The c.127C $>\mathrm{T}$ mutation in APOE-Kyoto leads to the amino acid substitution of Cys (TGC) for Arg (CGC) at codon 25.,4,18,23 The c.C127 is in the $\mathrm{CpG}$ dinucleotide and can be a target for methylation at the 5 carbon, yielding 5-methylcytosine, and be deaminated to produce thymine. ${ }^{24}$ As for APOE-Tokyo-Maebashi, the mutation is a deletion of 9-bp, ${ }^{1,19,23}$ with a 5-bp (CTGCG) overlapping sequence at the breakpoint, suggesting that it is a microhomology-mediated deletion. The frequencies of APOE-Kyoto and APOE Tokyo-Maebashi are likely because of the mutations at hot spots.

Recently, therapy with intensive lipid-lowering agents was reported to be effective. ${ }^{5,6,10,11}$ The establishment of effective therapy encourages early detection, which requires epidemiologic information on mutation. The first LPG patient with APOE-Sendai was from Yamagata prefecture, ${ }^{2}$ and more than 20 patients have now been detected in the eastern part of Japan. ${ }^{1-6}$ We recently identified five families with LPG (families 1, 2, 3, 6 and 9 in Figure 2) in Yamagata prefecture and detected two non-symptomatic healthy carriers of APOE-Sendai in two families (families 1 and 2). We had expected there to be twice as many or more asymptomatic carriers compared with LPG patients because of its low penetrance, but we could not find any carriers in control subjects and patients receiving hemodialysis in Yamagata prefecture. These findings indicate that the APOESendai mutation is common in Japanese patients with LPG; however, it is very rare in the general population.

For the pathogenesis of LPG, individuals carrying APOE mutations do not always have LPG (low penetrance), suggesting that additional genetic and/or epigenetic factors are likely to be involved in the onset 
of the disease. ${ }^{1}$ In our study, the haplotype of the counterpart APOE allele in the LPG patients was not identical or similar and some patients had the same haplotype of the counterpart allele as that of asymptomatic carriers (Figure 2). These results suggest that the counterpart $A P O E$ allele is not likely to be associated with the pathogenesis. Further study is required to clarify the pathogenesis of LPG, including the involvement of other genetic and/or epigenetic factors.

\section{CONFLICT OF INTEREST}

The authors declare no conflict of interest.

\section{ACKNOWLEDGEMENTS}

We thank Professor Hitoshi Nakashima (Fukuoka University School of Medicine) for his critical reading of the manuscript. We thank Dr Kazuhiro Akaba, Dr Tomoaki Shimokaze, Dr Toshiyuki Kimura, Dr Shinichi Sasa, Dr Tomomi Honma, Dr Takao Niki, Dr Junko Nagasawa, Dr Masahiko Okada, Dr Toshiyuki Shinozaki, Dr Michio Nagaoka, Dr Junichiro Nagasawa, Dr Hiroshi Onuma, Dr Akira Kajinuma, Dr Hiroshi Abiko, Dr Ken Ataka, Dr Kazutaka Satoh, Dr Moriyuki Nishina, Dr Sunao Izuha, Dr Toshiyuki Takahashi, Dr Masato Hoshikawa, Dr Hiroki Niino and Ms. Kumiko Mogami for their cooperation in providing clinical information and samples for the study. This study was supported in part by a Grant-in-aid for Scientific Research from the Ministry of Education, Culture, Sports, Science and Technology of Japan (No. 23791149) and a Grant-in-aid for Child Health from Yamagata Prefecture.

1 Saito, T., Matsunaga, A. \& Oikawa, S. Impact of lipoprotein glomerulopathy on the relationship between lipids and renal diseases. Am. J. Kidney Dis 47, 199-211 (2006).

2 Saito, T., Sato, H., Kudo, K., Oikawa, S., Shibata, T., Hara, Y. et al. Lipoprotein glomerulopathy: glomerular lipoprotein thrombi in a patient with hyperlipoproteinemia. Am. J. Kidney Dis. 13, 148-153 (1989).

3 Oikawa, S., Matsunaga, A. Saito, T. Sato, H. Seki, T., Hoshi, K et al. Apolipoprotein E Sendai (arginine $145 \rightarrow$ proline): a new variant associated with lipoprotein glomerulopathy. J. Am. Soc. Nephrol. 8, 820-823 (1997).

4 Tsimihodimos, V. \& Elisaf, M. Lipoprotein glomerulopathy. Curr. Opin. Lipidol. 22 262-269 (2011).

5 Matsunaga, A., Furuyama, M., Hashimoto, T., Toyota, K., Ogino, D. \& Hayasaka, K. Improvement of nephrotic syndrome by intensive lipid-lowering therapy in a patient with lipoprotein glomerulopathy. Clin. Exp. Nephrol 13, 659-662 (2009).

6 Hashimoto, T., Toyota, K., Ogino, D., Matsunaga, A. \& Hayasaka, K. A second pediatric patient with lipoprotein glomerulopathy carrying a heterozygous APOE-Sendai mutation. Jpn. J. Pediatr. Nephrol. 24, 218-223 (2011).
7 Tokura, T., Itano, S., Kobayashi, S., Kuwabara, A., Fujimoto, S., Horike, H. et al. A novel mutation ApoE2 Kurashiki (R158P) in a patient with lipoprotein glomerulopathy. J. Atheroscler. Thromb. 18, 536-541 (2011).

8 Mitani, A., Ishigami, M., Watase, K., Minakata, T. \& Yamamura, T. A nove apolipoprotein E mutation, ApoE Osaka (Arg158 Pro), in a dyslipidemic patient with lipoprotein glomerulopathy. J. Atheroscler. Thromb. 18, 531-535 (2011).

9 Liao, M. T., Tsai, I. J., Cheng, H. T., Lin, W. C., Chang, Y. W., Lin, Y. H. et al. A rare cause of childhood-onset nephrotic syndrome: lipoprotein glomerulopathy. Clin. Nephrol. 78, 237-240 (2012).

10 leiri, N., Hotta, O. \& Taguma, Y. Resolution of typical lipoprotein glomerulopathy. Am. J. Kidney. Dis. 41, 244-249 (2003).

11 Arai, T., Yamashita, S., Yamane, M., Manabe, N., Matsuzaki, T., Kiriyama, K. et al. Disappearance of intraglomerular lipoprotein thrombi and marked improvement of nephrotic syndrome by bezafibrate treatment in a patient with lipoprotein glomerulopathy. Atherosclerosis 169, 293-299 (2003).

12 Nomachi, S., Tagami, Y., Nakazawa, E., Midzushima, Y., Ozaki, T., Fujita, K. et al. Genetic diagnostic scheme for adult-onset type 2 citrullinemia using dried blood samples. Annu. Rep. Sapporo City Institute Public Health 30, 41-46 (2003).

13 Fullerton, S. M., Clark, A. G., Weiss, K. M., Nickerson, D. A., Taylor, S. L., Stengârd, J. H. et al. Apolipoprotein $\mathrm{E}$ variation at the sequence haplotype level: implications for the origin and maintenance of a major human polymorphism. Am. J. Hum. Genet. 67 881-900 (2000)

14 Sam, R., Wu, H., Yue, L., Mazzone, T., Schwartz, M. M., Arruda, J. A. et al. Lipoprotein glomerulopathy: a new apolipoprotein $\mathrm{E}$ mutation with enhanced glomerular binding. Am. J. Kidney Dis. 47, 539-548 (2006).

15 Luo, B., Huang, F., Liu, Q., Li, X., Chen, W., Zhou, S. F. et al. Identification of apolipoprotein E Guangzhou (arginine 150 proline), a new variant associated with lipoprotein glomerulopathy. Am. J. Nephrol. 28, 347-353 (2008).

16 Bomback, A. S., Song, H., D'Agati, V. D., Cohen, S. D., Neal, A., Appel, G. B. et al. A new apolipoprotein $\mathrm{E}$ mutation, apoE Las Vegas, in a European-American with lipoprotein glomerulopathy. Nephrol. Dial. Transplant. 25, 3442-3446 (2010).

17 Russi, G., Furci, L., Leonelli, M., Minnei, F., Magistroni, R., Romano, N. et al. Lipoprotein glomerulopathy treated with LDL-apheresis (Heparin-induced extracorporeal lipoprotein precipitation system.): a case report. J. Med. Case Rep 3, 9311 (2009).

18 Matsunaga, A., Sasaki, J., Komatsu, T., Kanatsu, K., Tsuji, E., Moriyama, K. et al. A novel apolipoprotein E mutation, E2 (Arg25Cys), in lipoprotein glomerulopathy. Kidney Int. 56, 421-427 (1999).

19 Ogawa, T., Maruyama, K., Hattori, H., Arai, H., Kondoh, I., Egashira, T. et al. A new variant of apolipoprotein $\mathrm{E}$ (apo $\mathrm{E}$ Maebashi) in lipoprotein glomerulopathy. Pediatr. Nephrol. 14, 149-151 (2000).

20 Rovin, B. H., Roncone, D., Mckinley, A., Nadasdy, T., Korbet, S. M. \& Schwartz, M. M. APOE Kyoto mutation in European Americans with lipoprotein glomerulopathy. N. Eng/. J. Med. 357, 2522-2524 (2007).

21 Foster, K., Matsunaga, A., Matalon, R., Saito, T., Gallo, G., Agati, V. D. et al. A rare cause of posttransplantation nephrotic syndrome. Am. J. Kidney Dis. 45, 1132-1138 (2005).

22 Konishi, K., Saruta, T., Kuramochi, S., Oikawa, S., Saito, T., Han, H. et al. Association of a novel 3-amino acid deletion mutation of apolipoprotein $E$ (Apo E Tokyo) with lipoprotein glomerulopathy. Nephron 83, 214-218 (1999).

23 Han, J., Pan, Y., Chen, Y., Li, X., Xing, G., Shi, J. et al. Common apolipoprotein E gene mutations contribute to lipoprotein glomerulopathy in China. Nephron Clin. Pract. 114, c260-c267 (2010).

24 Frommer, M., McDonald, L. E., Millar, D. S., Collis, C. M., Watt, F., Grigg, G. W. et al. A genomic sequencing protocol that yields a positive display of 5-methylcytosine residues in individual DNA strands. Proc. Natl Acad. Sci. USA 89, 1827-1831 (1992)

Supplementary Information accompanies the paper on Journal of Human Genetics website (http://www.nature.com/jhg) 